\title{
Interplay between SUMOylation and NEDDylation regulates RPL11 localization and function
}

\author{
Ahmed Motiam, Santiago Vidal Freire, Carlos de la Cruz-Herera, Sabela da \\ Silva -Álvarez, Maite Baz-Martínez, Anxo Vidal, Manuel Rodriguez, Dimitris \\ Xirodimas, Ana Carvalho, Hans Beck, et al.
}

\section{To cite this version:}

Ahmed Motiam, Santiago Vidal Freire, Carlos de la Cruz-Herera, Sabela da Silva -Álvarez, Maite Baz-Martínez, et al.. Interplay between SUMOylation and NEDDylation regulates RPL11 localization and function. FASEB Journal, Federation of American Society of Experimental Biology, 2019, 10.1096/fj.201800341RR . hal-03018811

\section{HAL Id: hal-03018811 https://hal.archives-ouvertes.fr/hal-03018811}

Submitted on 23 Nov 2020

HAL is a multi-disciplinary open access archive for the deposit and dissemination of scientific research documents, whether they are published or not. The documents may come from teaching and research institutions in France or abroad, or from public or private research centers.
L'archive ouverte pluridisciplinaire HAL, est destinée au dépôt et à la diffusion de documents scientifiques de niveau recherche, publiés ou non, émanant des établissements d'enseignement et de recherche français ou étrangers, des laboratoires publics ou privés. 


\section{Interplay between SUMOylation and NEDDylation of RPL11 impacts on p53 activation/Competition between SUMO and NEDD8 to conjugate to RPL11 controls p53.}

Ahmed EI Motiam ${ }^{1}$, Santiago Vidal Freire ${ }^{1}$, Carlos F de la Cruz-Herera ${ }^{2,3}$, Sabela Da Silva-Álvarez ${ }^{4}$, Maite Baz-Martínez ${ }^{1}$, Anxo Vidal ${ }^{1}$, Manuel S Rodríguez ${ }^{5,6}$, Dimitris P. Xirodimas $^{7}$, Ana Sofía Carvalho ${ }^{8}$, Hans Christian Beck ${ }^{9}$, Rune Mathiessen ${ }^{8}$, Manuel Collado $^{4}$, Carmen Rivas ${ }^{1,2^{*}}$

1 Centro de Investigación en Medicina Molecular (CIMUS), Universidade de Santiago de Compostela, Instituto de Investigaciones Sanitarias (IDIS), Santiago de Compostela, Spain.

2 Departamento de Biología Molecular y Celular, Centro Nacional de BiotecnologíaCSIC, Madrid, Spain.

3 Department of Molecular Genetics, University of Toronto, 1 Kings College Circle, Toronto M5S 1A8, Canada.

4 Instituto de Investigación Sanitaria de Santiago de Compostela (IDIS), Complexo Hospitalario Universitario de Santiago de Compostela (CHUS), SERGAS, Santiago de Compostela, Spain.

5 Advanced Technology Institute in Life Sciences (ITAV) CNRS-USR3505, Toulouse, France.

6 University of Toulouse III-Paul Sabatier, Toulouse, France.

7 CRBM, CNRS, Univ. Montpellier, UMR5237, Montpellier, Cedex 5, France.

8 CEDOC, Fac Ciencias Médicas, Universidade Nova de Lisboa, Lisboa, Portugal.

9 Odense University Hospital, Centre for Clinical Proteomics. Odense, Denmark.

Running title: Regulation of the RPL11-Mdm2-p53 pathway by SUMO

*Corresponding author: crivas@cnb.csic.es 


\begin{abstract}
The ribosomal protein RPL11 integrates different types of stress into a p53-mediated response. Here we analyzed the impact of the ubiquitin-like protein SUMO on the RPL11-Mdm2-p53 signaling. We show that SUMO1 and SUMO2 covalently modify RPL11. Interestingly, mutation of all lysine residues in RPL11 did not abolish SUMOylation, suggesting that this conjugation occurs through an alternative noncanonical SUMOylation pathway. We find that SUMO negatively modulates the conjugation of the ubiquitin-like protein NEDD8 to RPL11 and promotes the translocation of the ribosomal protein outside of the nucleoli. Moreover, the SUMO conjugating enzyme, Ubc9, is required for RPL11-mediated activation of p53. SUMOylation of RPL11 is triggered by ribosomal stress as well as by ARF upregulation. Collectively, our data identify SUMO protein conjugation to RPL11 as a new regulator of the p53-mediated cellular response to different types of stress, and reveal a previously unknown SUMO-NEDD8 interplay.
\end{abstract}




\section{INTRODUCTION}

The Mdm2-p53 pathway plays an essential role in coordinating cellular responses to stress. Under normal physiological conditions, p53 is maintained at low levels by its interaction with the E3 ubiquitin ligase Mdm2 ${ }^{1-5}$. Upon a cellular insult, Mdm2 is inhibited leading to p53 stabilization and activation. Several mechanisms have been shown to regulate the activity and levels of $\mathrm{Mdm} 2$. One of the $\mathrm{Mdm} 2$ regulators is the ribosomal RPL11 protein. In response to different types of stress, RPL11 is released from the nucleoli to the nucleoplasm where it interacts with Mdm2, inhibits its ubiquitin ligase activity, and induces the upregulation and activation of p53. Therefore, RPL11 is generally considered as a key player in coordinating the p53 response to nucleolar stress and it has been proposed as one of the factors involved in activating p53 under oncogenic and replicative stresses ${ }^{6-11}$. Several proteins have been reported to influence the RPL11-Mdm2 interaction. The covalent modification of RPL11 with the ubiquitinlike protein NEDD8 promotes its nucleolar localization, and controls the signaling of RPL11 to p53 in response to ribosomal stress ${ }^{12}$. In contrast, the interaction of the tumor suppressor ARF with RPL11, enhanced in response to either oncogenic or nucleolar stress, positively modulates the RPL11-Mdm2 binding, leading to the inhibition of p53 ubiquitination and to p53 activation ${ }^{7}$. How the activities of the regulatory proteins are coordinated on RPL11 to regulate p53 is currently unknown but it is clear that posttranslational modification of the RPL11-Mdm2-p53 pathway components may play an important role in such regulation.

SUMOylation is a post-translational modification with an important role in maintaining normal cell and tissues homeostasis. It consists in the covalent conjugation of small ubiquitin related modifiers (SUMO) to target proteins through a reversible and dynamic enzymatic process. This modification may impact different properties of proteins but it 
mainly regulates protein-protein interactions ${ }^{13}$. In mammals there are several SUMO isoforms that show some differences. SUMO2/3 share 97\% sequence identity and show around 50\% amino acid identity with SUMO1. SUMO2/3 can form SUMO chains whereas SUMO1 lacks a SUMOylation motif. Moreover, SUMO2/3 is present in greater abundance than SUMO1 and is predominantly involved in cell stress responses including oxidative stress, osmotic stress, heat shock or virus infection ${ }^{14-20}$.

Here we evaluated whether SUMO, by conjugation to RPL11, can regulate the Mdm2p53 pathway. Our results show that RPL11 can be modified by SUMO1 and SUMO2 in vitro and in vivo. SUMO negatively regulates the conjugation of NEDD8 to RPL11, favors the release of the ribosomal protein outside of the nucleoli and its conjugating enzyme Ubc9 is required for the RPL11 activation of p53. RPL11 SUMOylation is promoted by ribosomal stress or after upregulation of the tumor suppressor ARF. Interestingly, the SUMOylation of a lysine-KO RPL11 mutant protein suggests that SUMO conjugation to RPL11 occurs through an alternative non-canonical SUMOylation pathway.

\section{RESULTS}

\section{Ribosomal protein RPL11 is modified by SUMO}

To evaluate whether RPL11 can be modified by SUMO, we carried out in vitro SUMOylation assays using in vitro translated $\left[{ }^{35} \mathrm{~S}\right]$ methionine-labelled RPL11 protein as a substrate. In vitro translated myc-RPL11 protein was detected as a band of around $28 \mathrm{kDa}$ molecular weight, as expected. When the reaction was incubated with SUMO1 we detected at least an additional higher molecular weight band of around $43 \mathrm{kDa}$ (Figure 1A, left panel). When the reaction was incubated with SUMO2, we observed at least two additional higher molecular weight bands: one of around $43 \mathrm{kDa}$ and a fainter 
band of around $58 \mathrm{kDa}$ (Figure 1A, left panel). These results indicated that RPL11 is modified by SUMO1 and SUMO2 in vitro. To further probe that the bands correspond to RPL11-SUMO conjugates, we incubated RPL11-SUMO1 or RPL11-SUMO2 protein with the recombinant SUMO-specific protease SENP1. The high molecular weight bands detected when the protein was incubated with SUMO1 or SUMO2 disappeared after incubation of the reactions with SENP1 (Figure 1A, right panel). All together, these results demonstrate that RPL11 is modified by SUMO1 and SUMO2 in vitro. Then, in order to determine whether RPL11 also conjugates to SUMO within the cell, HEK-293 cells were co-transfected with myc-RPL11 together with pcDNA, Ubc9 and His6-SUMO1 or His6-SUMO2. At $48 \mathrm{~h}$ after transfection both whole protein extracts and Histidine tagged proteins purified under denaturing conditions using nickel beads were analyzed by Western-blot using anti-myc antibody. Western-blot analysis of the purified extracts revealed bands of the expected size corresponding to RPL11-SUMO1 and RPL11-SUMO2 only in those cells co-transfected with His6-SUMO1 and His6SUMO2, respectively, indicating that RPL11 is modified by SUMO1 and SUMO2 in the transfected cells (Figure 1B). We then decided to evaluate the SUMOylation of endogenous RPL11 protein. HEK-293 cells were transfected with pcDNA, Ubc9 and His6-SUMO1 or His6-SUMO2. Western-blot analysis of the whole protein extracts using anti-RPL11 antibody revealed a band of around $20 \mathrm{kDa}$, as expected (Figure 1C). Purification of the Histidine tagged proteins under denaturing conditions and Westernblot analysis using anti-RPL11 antibody revealed the presence of the bands corresponding with RPL11-SUMO1 and RPL11-SUMO2 bands only in those cells transfected with His6-SUMO1 or His6-SUMO2, respectively, demonstrating that endogenous RPL11 protein is also modified by SUMO1 and SUMO2 (Figure 1C). All 
together these results demonstrate that RPL11 is modified by SUMO1 and SUMO2 in vitro and in cells.

\section{SUMO conjugates to a non-lysine residue in RPL11}

In silico analysis of the RPL11 amino acid sequence using SUMOsp prediction program pointed to lysine residue K78 as a type I SUMOylation site with the highest probability to be involved in SUMO conjugation and to lysine K158 as a less probable SUMO conjugating site. However, SUMOylation analysis of RPL11 protein mutated in these lysine residues revealed no differences in the SUMO conjugation relative to the WT protein. Then, we decided to evaluate the SUMOylation of a set of plasmid constructs expressing mutants of RPL11 in different lysine residues simultaneously as well as of a mutant in which all lysine residues are mutated (RPL11-KO) (Figure 2A). In vitro SUMOylation experiments revealed no significant differences in SUMO1 conjugation between the RPL11 proteins evaluated (Figure 2B), suggesting that SUMO conjugates to a non-lysine residue in RPL11. To verify this result in cells, we co-transfected HEK293 cells with HA-RPL11-WT or HA-RPL11-KO together with pcDNA or Ubc9 and His6-SUMO2. Western-blot analysis of the Histidine tagged proteins purified under denaturing conditions with anti-HA antibody revealed that SUMO2 modifies the lysineKO RPL11 mutant in a covalent manner (Figure 2C), suggesting that RPL11 can be SUMOylated through a previously undescribed mechanism.

\section{Interplay between SUMO and NEDD8 conjugation to RPL11}

RPL11 can be conjugated to the ubiquitin-like protein NEDD8, with only mutation of all lysine residues in RPL11 resulting in a decrease in NEDDylation ${ }^{12}$. Therefore, we speculated that SUMO might compete with NEDD8 for interaction with RPL11. To evaluate this hypothesis, we first co-transfected HEK-293 cells with myc-RPL11 together with pcDNA, His6-NEDD8 and pcDNA or untagged SUMO1. At $48 \mathrm{~h}$ after 
transfection both whole protein extracts and Histidine tagged proteins purified under denaturing conditions were analyzed by Western-blot with anti-myc antibody. Analysis of the purified extracts revealed the appearance of bands corresponding to RPL11NEDD8 protein exclusively in those cells transfected with His6-NEDD8, as expected (Figure 3A). Interestingly, we observed a clear decrease in the levels of NEDDylated RPL11 protein in those cells co-transfected with SUMO1 (Figure 3A), indicating that upregulation of SUMO1 downmodulates the NEDDylation of RPL11. We then cotransfected HEK-293 cells with myc-RPL11 together with pcDNA, His6-NEDD8 and pcDNA, Ubc9, His6-SUMO2 and pcDNA or Ubc9, His6-SUMO2 and His6-NEDD8 and $48 \mathrm{~h}$ after transfection, Histidine tagged proteins were purified under denaturing conditions. Western-blot analysis of the purified proteins using anti-myc antibody revealed the appearance of bands corresponding to RPL11-SUMO2 and RPL11NEDD8 in those cells transfected with His6-SUMO2 or His6-NEDD8, respectively (Figure 3B). When we co-transfected His6-SUMO2 together with His6-NEDD8 we consistently observed reduced levels of the bands corresponding with NEDDylated RPL11 protein (Figure 3B), indicating that SUMO2 negatively regulates the conjugation of RPL11 to NEDD8. Then, we decided to study whether treatment with SUMOylation or NEDDylation inhibitors had an effect on the conjugation of RPL11 to NEDD8 or SUMO2. We first co-transfected HEK-293 cells with myc-RPL11 together with pcDNA, His6-NEDD8 or Ubc9 and His6-SUMO2 and $36 \mathrm{~h}$ after transfection cells were treated or not the SUMOylation inhibitor ginkgolic acid (GA). Western-blot analysis of the whole protein extracts using anti-myc antibody revealed that the levels of RPL11-SUMO2 protein detected in cells transfected with SUMO2 decreased after treatment with GA (Figure 3C, upper panel). In contrast, we observed a clear increase in the levels of NEDDylated RPL11 protein in the presence of the SUMOylation inhibitor 
(Figure 3C, lower panel). Finally, HEK-293 cells were transfected as described above and $36 \mathrm{~h}$ after transfection, cells were treated with the NEDDylation inhibitor MLN4924 for $4 \mathrm{~h}$. Western-blot analysis of the whole cell protein extracts using antimyc antibody revealed the appearance of NEDDylated-RPL11 bands in the cells transfected with NEDD8, whose intensity decreased after treatment with MLN4924, as previously reported ${ }^{21}$ (Figure 3D). In contrast, treatment of cells with MLN4929 induced a clear upregulation in the levels of RPL11-SUMO2 protein (Figure 3D). All together these results demonstrate that there is an interplay between SUMOylation and NEDDylation of RPL11.

As we show here for SUMO, it has been previously reported that NEDD8 can still conjugate to RPL11-KO ${ }^{12}$. Therefore we decided to evaluate whether SUMO2 also regulates the conjugation of NEDD8 to RPL11-KO. We co-transfected HEK-293 cells with HA-RPL11-WT or HA-RPL11-KO together with pcDNA, His6-NEDD8 and pcDNA, Ubc9, His6-SUMO2 and pcDNA or Ubc9, His6-SUMO2 and His6-NEDD8 and $48 \mathrm{~h}$ after transfection, Histidine tagged proteins were purified under denaturing conditions. Western-blot analysis of the purified proteins using anti-HA antibody revealed the appearance of bands corresponding to RPL11-SUMO2 and RPL11NEDD8 in those cells transfected with His6-SUMO2 or His6-NEDD8, respectively (Figure 4). We also observed a clear reduction in the intensity of the NEDDylated and SUMOylated RPL11 bands in those cells transfected with RPL11-KO, as expected. Cotransfection of His6-SUMO2 together with His6-NEDD8 clearly induced a reduction in the levels of the bands corresponding with NEDDylated RPL11-WT protein, as shown above. However, we did not observe a negative effect of SUMO2 on the NEDDylation of RPL11-KO (Figure 4). These results indicate that SUMO2 competes with NEDD8 
for common amino acid residues in RPL11-WT. However, SUMO2 and NEDD8 do not compete for the same residues on RPL11-KO.

\section{Ribosomal stress promotes the modification of RPL11 by SUMO2}

It has been reported that upon nucleolar stress RPL11 is de-NEDDylated ${ }^{12}$. Our results indicate that there is an antagonistic relationship between SUMOylation and NEDDylation of RPL11, therefore we decided to evaluate whether the SUMOylation of RPL11 was altered in response to nucleolar stress. U2OS cells were co-transfected with myc-RPL11 and pcDNA or Ubc9 and His6-SUMO2 and $36 \mathrm{~h}$ after transfection, cells were treated with a low dose of actinomycin D for the indicated times. As shown in Figure 5, the Western-blot analysis of the Histidine tagged proteins purified in denaturing conditions using anti-myc antibody revealed an increase in the levels of RPL11-SUMO2 protein in response to actinomycin D, indicating that nucleolar stress promotes SUMO2 modification of RPL11.

\section{SUMO2 promotes the release of RPL11 from the nucleolus and the SUMO ligase} Ubc9 is required for the activation of p53 in response to RPL11 upregulation. NEDD8 regulates the nucleolar localization of RPL11 ${ }^{12}$. Therefore, we decided to evaluate the effect of SUMO on the subcellular localization of RPL11. We cotransfected MCF7 cells with myc-RPL11 together with pcDNA or His6-SUMO2 and 48 $\mathrm{h}$ after transfection, cells were analyzed by immunofluorescence using anti-myc and anti-SUMO2 antibodies. RPL11 was mainly localized in the nucleolus of those cells cotransfected with RPL11 and pcDNA, as expected (Figure 6A). However, RPL11 was detected in the nucleoplasm of those cells expressing high levels of SUMO2, indicating that SUMO promotes the release of RPL11 from the nucleolus to the cell nucleoplasm (Figure 6A). 
Transfection of RPL11 led to stabilization of p53 and the induction of p53-dependent cell cycle arrest ${ }^{8,11}$. We therefore examined whether downmodulation of SUMO conjugation has an impact on the RPL11 mediated p53 stabilization or activation. U2OS cells were first transfected with Ubc9 siRNA (siUbc9) or scramble siRNA (siC) for 48 $\mathrm{h}$, then cells were transfected or not with RPL11 for $24 \mathrm{~h}$, and finally they were treated with cycloheximide $(\mathrm{CHX})$ for the indicated times. The levels of p53 at each time after CHX treatment were evaluated by Western-blot analysis. We did not observe an alteration in the stability of p53 after Ubc9 downmodulation in those cells untransfected with RPL11 (Figure 6B, upper panel). Transfection of RPL11 clearly increased the stability of p53, as expected (Figure 6B, lower panel). We also observed a clear reduction in the stability of p53 in those cells with lower levels of Ubc9 (Figure 6B, lower panel). All together these results suggest that SUMOylation contributes for p53 stabilization after RPL11 overexpression.

We then evaluated the effect of Ubc9 downmodulation on the cell cycle arrest induced after ectopic expression of RPL11. U2OS cells transfected with siUbc9 or siC as described above were co-transfected with pcDNA or RPL11 together with a plasmid expressing GFP-F for gating the positively transfected cells. After $24 \mathrm{~h}$, the cell cycle distribution was determined by flow cytometry analysis. We observed a significant reduction in the percentage of cells in S-phase in the cells co-transfected with siC and RPL11 relative to the percentage detected in the cells transfected with siC and pcDNA (Figure 6C), as expected ${ }^{11}$. However, we did not observe a significant reduction in the percentage of cells in S-phase when RPL11 was introduced in siUbc9 transfected cells (Figure 6C), suggesting that SUMOylation is required for the activation of p53 in response to RPL11 overexpression. 


\section{SUMO2 modification of RPL11 is induced by ARF}

RPL11 interacts with ARF and it is a mediator in ARF regulated p53 activation ${ }^{7,11}$. In addition, ARF has been reported to promote the SUMOylation of several proteins to which ARF binds ${ }^{22,23}$. Therefore, we decided to evaluate whether ARF also promotes RPL11 SUMOylation. U2OS cells (ARF null) were co-transfected with myc-RPL11 together with pcDNA or Ubc9 and His6-SUMO2, and in the presence or absence of GFP-ARF. At $48 \mathrm{~h}$ after transfection whole protein extracts and Histidine tagged proteins purified under denaturing conditions were evaluated by Western-blot using anti-myc antibody. As shown in Figure 7A (upper panel), expression of ARF clearly upregulated the levels of RPL11-SUMO2 protein. Similar results were observed when the experiment was carried out in the p53-null H1299 cell line (Figure 7A, lower panel). Moreover, since our results indicated that SUMO negatively regulates the NEDDylation of RPL11, we also decided to evaluate whether the ectopic expression of ARF has also an impact on RPL11 NEDDylation. U2OS cells were co-transfected with myc-RPL11 and pcDNA or His6-NEDD8, and in the presence or absence of GFP-ARF. At $48 \mathrm{~h}$ after transfection, the whole cell protein extracts as well as the Histidine tagged purified proteins were analyzed by Western-blotting. NEDDylation of RPL11 was dramatically downmodulated after expression of ARF (Figure 7B). Interestingly, a global downmodulation of NEDD8 conjugation was also observed (Figure 7B).

\section{DISCUSSION}

Here we identified SUMO as a regulator of the RPL11-Mdm2-p53 pathway. Our data reveal that RPL11 can be modified by SUMO1 and SUMO2 in vivo and in cells. We attempted to create an RPL11 SUMOylation mutant. However, our results revealed that even mutation of all the lysine residues in RPL11 did not abolish SUMOylation, 
suggesting the existence of a non-canonical SUMOylation pathway, as it has been already reported for ubiquitin ${ }^{29}$. Similarly to what we observed with SUMO, NEDD8 conjugation to RPL11 was only reduced after mutation of all lysine residues in RPL11 and a residual modification of this mutant was still reported ${ }^{12}$. Therefore, we speculated that NEDD8 and SUMO might compete for conjugation to RPL11. Our results showed that NEDDylation of RPL11 decreases after SUMO overexpression and increases after treatment with the SUMOylation inhibitor GA, indicating that SUMO negatively regulates the NEDDylation of RPL11. Although SUMOylation of RPL11 was positively modulated after NEDDylation inhibitor treatment, suggesting that NEDD8 also negatively regulates the SUMOylation of RPL11, this modification was not reduced by NEDD8 overexpression. We speculate that the RPL11 stabilization or nucleolar localization promoted by NEDD8 ${ }^{12}$ may have a positive impact on RPL11 SUMOylation. Different points of crosstalk may occur between SUMOylation and NEDDylation, including competition for common amino acid residues or regulation of the NEDD8 conjugation machinery by SUMO. In contrast to the competition observed between SUMO and NEDD8 conjugation on RPL11-WT, SUMO2 did not compete with NEDD8 to conjugate to RPL11-KO, suggesting that a regulation of the NEDDylation machinery by SUMO is not involved in the SUMO-NEDD8 interplay on RPL11. Accordingly with the existence of an antagonistic relationship between NEDDylation and SUMOylation of RPL11, we observed that the SUMO2 modification of RPL11 was promoted by ribosomal stress, a stimuli that has been shown to trigger the deNEDDylation of RPL11 ${ }^{12}$. Moreover, and in agreement with an inverse correlation between these two post-translational modifications, we also observed that overexpression of SUMO2 promoted the translocation of RPL11 from the nucleolus to 
the nucleoplasm. So far, this is the first description of an interplay between SUMOylation and NEDDylation.

As a result of nucleolar stress or after ectopic expression of RPL11, the ribosomal protein binds Mdm2 and promotes p53 activation ${ }^{6,8,11,24,25}$. Our data showed that the SUMO ligase Ubc9 was required for the stabilization and activation of p53 in response to RPL11 overexpression. SUMO has been previously shown to modulate several components of the RPL11-Mdm2-p53 pathway, including p53 ${ }^{26,27}$ or Mdm2 ${ }^{28}$. Therefore, we cannot discard that the negative effect of Ubc9 downmodulation on the stability or activity of p53 we observed was not due to SUMOylation inhibition of other factors and not of RPL11.

Although RPL11 is mainly known as a key protein in the control of p53 activation in response to ribosomal stress, recent reports demonstrate that RPL11 is also required for oncogenic or replicative stress-induced activation of p53 ${ }^{9}$ and for activation of p53 by ARF ${ }^{10}$. The molecular mechanisms underlying the RPL11 mediated p53 activation upon replicative or oncogenic stress are not known. One proposed explanation is that the increase in ARF levels resulting from replicative or oncogenic stress induces ribosomal stress resulting in RPL11 suppression of Mdm2 ${ }^{7,9}$. We show here that upregulation of ARF triggers the SUMO2 modification of RPL11, leading us to propose that promotion of RPL11 SUMOylation by ARF may be a molecular link between the oncogenic or replicative stress and the ribosomal stress.

The ability of ARF to trigger SUMOylation of several cellular proteins and to enhance global SUMO conjugation has been previously reported $22,23,30,31$. The mechanism by which ARF increases SUMOylation is not clearly known. Here we show that upregulation of ARF leads also to a decrease in RPL11 NEDDylation and, importantly, in global NEDDylation. Further studies will be required to determine whether the 
downmodulation of global NEDD8 conjugation by ARF results from the upregulation in the SUMOylation mediated by the tumor suppressor.

\section{Experimental procedures}

Cell lines and reagents. Human embryonic kidney epithelial 293 (HEK-293), human p53-null lung non-small cell carcinoma H1299, and the p53-WT MCF7 breast cancer and U2OS osteosarcoma cells were cultured in complete medium (DMEM supplemented with $10 \%$ fetal bovine serum, $1 \%$ penicillin/streptomycin). The cells were transiently transfected using Xtreme-GENE siRNA (Roche) or PEI (Polysciences, Inc) transfection reagents, as suggested by the manufacturer. Actinomycin D, cycloheximide and Ginkgolic acid were from Sigma. NEDDylation inhibitor MLN4924 was from Millipore. Smart-pool small interfering RNAs against Ubc9 (siUbc9) and scramble small interfering RNA (siC) were purchased from Dharmacon.

Plasmids and antibodies. The myc-tagged RPL11 expression plasmid was previously described $^{11}$ (Addgene plasmid 20936). Flag-RPL11-WT, Flag-RPL11 in which all the lysine residues were mutated to arginine (Flag-RPL11-KO), and plasmids encoding RPL11 containing simultaneous mutated lysines were previously described ${ }^{12}$. HARPL11-WT as well as HA-RPL11-KO was generated by PCR amplification of RPL11WT or RPL11-KO and cloning into the pcMV5-HA vector. pcDNA3.1-SUMO1 plasmid was previously described ${ }^{32}$. pcDNA-His6-SUMO1, pcDNA-His6-SUMO2, and pcDNA-SV5-Ubc9 plasmids were previously described ${ }^{33,34}$. pcDNA-His6-NEDD8 plasmid was provided by Dr. Manuel S Rodríguez. A quick-change site-directed mutagenesis kit (Agilent) was used to make amino acid changes using Flag-RPL11 plasmid as template. All mutations were confirmed by sequencing. Anti-HA antibody was from Covance. Anti-SUMO1 antibody was from Cell Signaling. Anti-NEDD8 
[Y297] was from Abcam. Anti-p53, anti-Flag, and anti-GAPDH were from Santa Cruz Biotecnology. Anti-GFP was from Biolegend. Antibodies against RPL11 were purchased from Invitrogen and Abcam. Anti-myc tag antibodies were purchased from Santa Cruz and Cell Signaling. Antibodies against SUMO2 were from Invitrogen and Cell Signaling.

In vitro SUMOylation assay. In vitro SUMO conjugation assays were performed on $\left[{ }^{35} \mathrm{~S}\right]$ methionine-labeled in vitro-transcribed/translated proteins as described previously 35 using recombinant E1 (Biomol), Ubc9, and SUMO1 or SUMO2. The in vitro transcription/translation of proteins was performed by using $1 \mu \mathrm{g}$ of plasmid DNA and a rabbit reticulocyte-coupled transcription/ translation system according to the instructions provided by the manufacturer (Promega).

In vitro deSUMOylation assay In vitro deSUMOylation assay with recombinant GSTSENP1 (Biomol) was performed on RPL11-SUMO1 or RPL11-SUMO2 as described previously 36

Purification of His-tagged conjugates. The purification of His-tagged conjugates using $\mathrm{Ni}^{2+}{ }_{-} \mathrm{NTA}-$ agarose beads was performed as described previously ${ }^{37}$. Immunofluorescence staining. Immunofluorescence staining and confocal analysis were performed as described previously ${ }^{36}$. Mouse anti-myc antibody was used at a dilution of 1:200, rabbit anti-SUMO2 antibody (1:200) was obtained from Invitrogen. Secondary Alexa 488-conjugated, and Alexa 594-conjugated antibodies were obtained from Invitrogen. Analysis of the samples was carried out on a Leica TCS SP5 confocal laser microscope using simultaneous scans to avoid shift between the optical channels. Images were exported by use of Adobe Photoshop.

Cell cycle analysis. U2OS cells previously transfected with siUbc9 or siC were cotransfected with farnesylated GFP (GFP-F) and pcDNA or myc-RPL11. 24h after 
transfection cells were fixed, permeabilized, and stained in a solution containing propidium iodide, RNase A, and Triton X-100. Cells were analyzed for DNA content using a Becton Dickinson FACScalibur flow cytometer using FlowJo software. GFP positive cells were gated for cell cycle analysis.

Statistical analysis. For statistical analysis between control and different groups the Student's t test was applied. The significance level chosen for the statistical analysis was $\mathrm{p}<0.05$.

\section{Conflict of Interest}

The authors declare no competing financial interest.

\section{Acknowledgements}

We thank Dr Yue Xong who deposited RPL11 plasmid at Addgene. Funding at the laboratory of CR is provided by Ministry of Ecomony and Competitiveness and FEDER (BFU-2014-58530. AEM) is a fellowship of the Spanish FPI program.

\section{Figure legends}

Figure 1. RPL11 is modified by SUMO1 and SUMO2 in vitro and in vivo. A, In vitro translated ${ }^{35}$ S-labeled RPL11 was subjected to in vitro SUMOylation assay in the presence of SUMO1 or SUMO2 (left panel). SUMO1 or SUMO2-conjugated RPL11 protein was then incubated in the presence or absence of SENP1 as described in Material and Methods (right panel). Proteins were resolved by SDS-polyacrylamide gel electrophoresis and visualized by autoradiography. Arrows and arrowheads indicate the 
unmodified and SUMO conjugated RPL11 protein, respectively. B. HEK-293 cells were co-transfected with myc-RPL11 together with pcDNA, Ubc9 and His6-SUMO1 or Ubc9 and His6-SUMO2. Whole protein extracts and Histidine tagged purified proteins were analyzed by Western-blot using anti-myc antibody. Arrow and arrowheads indicate the unmodified and SUMO conjugated RPL11 protein, respectively. C, HEK293 cells were transfected with pcDNA, Ubc9 and His6-SUMO1 or Ubc9 and His6SUMO2. Whole protein extracts and Histidine tagged purified proteins were analyzed by Western-blotting using anti-RPL11 antibody. Arrowheads indicate the SUMO conjugated RPL11 protein. The position of a nonspecific band is indicated by an asterisk.

Figure 2. SUMO conjugates to a non-lysine residue in RPL11. A, Scheme showing the RPL11 lysine residues that have been changed to arginine in each mutant (marked with a bold line). B, In vitro translated ${ }^{35}$ S-labeled Flag-RPL11-WT or the indicated mutant proteins were subjected to in vitro SUMOylation assay in the presence of SUMO1. Proteins were resolved by SDS-polyacrylamide gel electrophoresis and visualized by autoradiography. Arrrowheads indicate the SUMO conjugated RPL11 protein. C, HEK-293 cells were co-transfected with HA-RPL11-WT or HA-RPL11-KO together with pcDNA, or Ubc9 and His6-SUMO2. Whole protein extracts and Histidine tagged purified proteins were analyzed by Western-blot using anti-HA antibody. Arrow and arrowheads indicate the unmodified and SUMO2 conjugated RPL11 protein, respectively

Figure 3. Interplay between SUMO and NEDD8 conjugation to RPL11. A, HEK293 cells were co-transfected with myc-RPL11 together with pcDNA, His6-NEDD8 or His6-NEDD8, Ubc9 and untagged SUMO1. At $48 \mathrm{~h}$ after transfection, Histidine tagged purified proteins were analyzed by Western-blot using anti-myc antibody. Empty 
arrowheads indicate the NEDD8 conjugated RPL11 protein. Whole protein extracts were analyzed with the indicated antibodies. B, HEK-293 cells were co-transfected with myc-RPL11 together with pcDNA, His6-NEDD8, Ubc9 and His6-SUMO2 or His6NEDD8, Ubc9 and His6-SUMO2. At $36 \mathrm{~h}$ after transfection, Histidine tagged purified proteins were analyzed by Western-blot using anti-myc antibody. Empty and filled in arrowheads indicate the NEDD8 and the SUMO2 conjugated RPL11 protein, respectively. Whole protein extracts were analyzed with the indicated antibodies. C, HEK-293 cells were co-transfected with myc-RPL11 together with pcDNA, His6NEDD8 or Ubc9 and His6-SUMO2. At $36 \mathrm{~h}$ after transfection, cells were treated or not with the SUMOylation inhibitor GA $(25 \mu \mathrm{M})$. At $4 \mathrm{~h}$ after treatment, whole protein extracts were analyzed by Western-blot using the indicated antibodies. Arrows indicated the unmodified RPL11 protein. Empty and filled in arrowheads indicate the NEDD8 and the SUMO2 conjugated RPL11 protein, respectively. D, HEK-293 cells were cotransfected with myc-RPL11 together with pcDNA, His6-NEDD8 or Ubc9 and His6SUMO2. At $36 \mathrm{~h}$ after transfection, cells were treated or not with the NEDDylation inhibitor MLN4924 $(1 \mu \mathrm{M})$. At $4 \mathrm{~h}$ after treatment, whole protein extracts were analyzed by Western-blot using the indicated antibodies. Empty and filled in arrowheads indicate the NEDD8 and the SUMO2 conjugated RPL11 protein, respectively.

Figure 4. SUMO2 does not compete with NEDD8 to modify RPL11-KO. HEK-293 cells were co-transfected with HA-RPL11-WT or HA-RPL11-KO together with pcDNA, His6-NEDD8, Ubc9 and His6-SUMO2 or His6-NEDD8, Ubc9 and His6SUMO2. At $36 \mathrm{~h}$ after transfection, whole protein extracts and Histidine tagged purified proteins were analyzed by Western-blot using anti-HA antibody. In addition, input extracts were analyzed by Western-blot with anti-NEDD8 and-SUMO2 antibody. 
Arrows indicate the unmodified RPL11 bands. Empty and filled in arrowheads indicate NEDD8 and SUMO conjugated RPL11 protein, respectively.

Figure 5. Ribosomal stress promotes RPL11 SUMOylation U2OS cells were cotransfected with myc-RPL11 together with Ubc9 and His6-SUMO2. At $36 \mathrm{~h}$ after transfection, cells were treated with low concentration of actinomycin $\mathrm{D}(5 \mathrm{nM})$. At the indicated times after treatment, Histidine tagged purified proteins were analyzed by Western-blot using anti-myc antibody. Whole protein extracts were analyzed with the indicated antibodies. Arrowheads indicate the SUMO2 conjugated RPL11 protein.

Figure 6. SUMO2 promotes the release of RPL11 from the nucleoli and the SUMO ligase Ubc9 is required for the activation of p53 in response to RPL11 upregulation. A, MCF7 cells were co-transfected with myc-RPL11 and pcDNA or SUMO2, as indicated. At $48 \mathrm{~h}$ after transfection cells were immunostained with antimyc and anti-SUMO2 antibodies. Subcellular localization of the expressed proteins was analyzed under a confocal microscope. Images were processed with Adobe Photoshop. B, U2OS cells were transfected with siC or siUbc9 for $48 \mathrm{~h}$. Then, cells were transfected with myc-RPL11 or pcDNA at $24 \mathrm{~h}$ after transfection cells were treated with cycloheximide $(\mathrm{CHX})(100 \mu \mathrm{g} / \mathrm{ml})$. At the indicated times after $\mathrm{CHX}$ treatment protein extracts were recovered and analyzed by Western-blotting using the indicated antibodies. C, U2OS cells were transfected with siC or siUbc9 for $48 \mathrm{~h}$. Then, cells were co-transfected with farnesylated GFP (GFP-F) and myc-RPL11 or pcDNA. At 24 $\mathrm{h}$ after transfection, cells were harvested, fixed, permeabilized and stained with propidium iodide. GFP positive cells were gated for cell cycle analysis. Mean percentage of cells in $\mathrm{S}$ phase from triplicates is shown. Error bars are standard deviation of triplicates. $* \mathrm{P}<0.005$, Student's test. 
Figure 7. SUMO2 modification of RPL11 is promoted by ARF. A, U2OS cells (upper panel) or H1299 cells (lower panel) were co-transfected with myc-RPL11 together with pcDNA or Ubc9 and His6-SUMO2 and in the presence or absence of GFP-ARF. At $48 \mathrm{~h}$ after transfection, Histidine tagged purified proteins were analyzed by Western-blot using anti-myc antibody. Arrowheads indicate the SUMO2-conjugated RPL11 protein. Whole protein extracts were analyzed with the indicated antibodies. B, U2OS cells were co-transfected with myc-RPL11 together with pcDNA or His6NEDD8 and in the presence or absence of GFP-ARF. At $48 \mathrm{~h}$ after transfection, Histidine tagged purified proteins were analyzed by Western-blot using anti-myc antibody. Empty arrowheads indicate the NEDD8-conjugated RPL11 protein. Whole protein extracts were analyzed with the indicated antibodies.

1 Haupt, Y., Maya, R., Kazaz, A. \& Oren, M. Mdm2 promotes the rapid degradation of p53. Nature 387, 296-299 (1997).

2 Honda, R., Tanaka, H. \& Yasuda, H. Oncoprotein MDM2 is a ubiquitin ligase E3 for tumor suppressor p53. FEBS Lett 420, 25-27 (1997).

3 Kruse, J. P. \& Gu, W. Modes of p53 regulation. Cell 137, 609-622 (2009).

4 Kubbutat, M. H., Jones, S. N. \& Vousden, K. H. Regulation of p53 stability by Mdm2. Nature 387, 299-303 (1997).

5 Oliner, J. D. et al. Oncoprotein MDM2 conceals the activation domain of tumour suppressor p53. Nature 362, 857-860 (1993).

6 Bhat, K. P., Itahana, K., Jin, A. \& Zhang, Y. Essential role of ribosomal protein L11 in mediating growth inhibition-induced p53 activation. Embo J 23, 24022412 (2004).

7 Dai, M. S. et al. Physical and functional interaction between ribosomal protein L11 and the tumor suppressor ARF. J Biol Chem 287, 17120-17129 (2012).

8 Lohrum, M. A., Ludwig, R. L., Kubbutat, M. H., Hanlon, M. \& Vousden, K. H. Regulation of HDM2 activity by the ribosomal protein L11. Cancer Cell 3, 577587 (2003).

9 Nishimura, K. et al. Perturbation of ribosome biogenesis drives cells into senescence through 5S RNP-mediated p53 activation. Cell Rep 10, 1310-1323 (2015). 
10 Sloan, K. E., Bohnsack, M. T. \& Watkins, N. J. The 5S RNP couples p53 homeostasis to ribosome biogenesis and nucleolar stress. Cell Rep 5, 237-247 (2013).

11 Zhang, Y. et al. Ribosomal protein L11 negatively regulates oncoprotein MDM2 and mediates a p53-dependent ribosomal-stress checkpoint pathway. Mol Cell Biol 23, 8902-8912 (2003).

12 Sundqvist, A., Liu, G., Mirsaliotis, A. \& Xirodimas, D. P. Regulation of nucleolar signalling to p53 through NEDDylation of L11. EMBO Rep 10, 11321139 (2009).

13 Wilkinson, K. A. \& Henley, J. M. Mechanisms, regulation and consequences of protein SUMOylation. Biochem J 428, 133-145 (2010).

14 de la Cruz-Herrera, C. F. et al. SUMOylation regulates AKT1 activity. Oncogene 34, 1442-1450 (2014).

15 Domingues, P. et al. Global Reprogramming of Host SUMOylation during Influenza Virus Infection. Cell Rep 13, 1467-1480 (2015).

16 Enserink, J. M. Sumo and the cellular stress response. Cell Div 10, 4 (2015).

17 Guo, C. \& Henley, J. M. Wrestling with stress: roles of protein SUMOylation and deSUMOylation in cell stress response. IUBMB Life 66, 71-77 (2014).

18 Saitoh, H. \& Hinchey, J. Functional heterogeneity of small ubiquitin-related protein modifiers SUMO-1 versus SUMO-2/3. J Biol Chem 275, 6252-6258 (2000).

19 Sramko, M., Markus, J., Kabat, J., Wolff, L. \& Bies, J. Stress-induced inactivation of the c-Myb transcription factor through conjugation of SUMO-2/3 proteins. J Biol Chem 281, $40065-40075$ (2006).

20 Zhou, W., Ryan, J. J. \& Zhou, H. Global analyses of sumoylated proteins in Saccharomyces cerevisiae. Induction of protein sumoylation by cellular stresses. J Biol Chem 279, 32262-32268 (2004).

21 Bailly, A. et al. The NEDD8 inhibitor MLN4924 increases the size of the nucleolus and activates p53 through the ribosomal-Mdm2 pathway. Oncogene 35, 415-426 (2016).

22 Chen, L. \& Chen, J. MDM2-ARF complex regulates p53 sumoylation. Oncogene 22, 5348-5357 (2003).

23 Xirodimas, D. P., Chisholm, J., Desterro, J. M., Lane, D. P. \& Hay, R. T. P14ARF promotes accumulation of SUMO-1 conjugated $(\mathrm{H}) \mathrm{Mdm} 2$. FEBS Lett 528, 207-211 (2002).

24 Dai, M. S. \& Lu, H. Inhibition of MDM2-mediated p53 ubiquitination and degradation by ribosomal protein L5. J Biol Chem 279, 44475-44482 (2004).

25 Dai, M. S. et al. Regulation of the MDM2-p53 pathway by ribosomal protein L11 involves a post-ubiquitination mechanism. J Biol Chem 281, 24304-24313 (2006).

26 Gostissa, M. et al. Activation of p53 by conjugation to the ubiquitin-like protein SUMO-1. Embo J 18, 6462-6471 (1999).

27 Rodriguez, M. S. et al. SUMO-1 modification activates the transcriptional response of p53. Embo J 18, 6455-6461 (1999).

28 Buschmann, T., Fuchs, S. Y., Lee, C. G., Pan, Z. Q. \& Ronai, Z. SUMO-1 modification of Mdm2 prevents its self-ubiquitination and increases Mdm2 ability to ubiquitinate p53. Cell 101, 753-762 (2000).

29 McDowell, G. S. \& Philpott, A. Non-canonical ubiquitylation: mechanisms and consequences. Int J Biochem Cell Biol 45, 1833-1842 (2013). 
30 Rizos, H., Woodruff, S. \& Kefford, R. F. p14ARF interacts with the SUMOconjugating enzyme Ubc9 and promotes the sumoylation of its binding partners. Cell Cycle 4, 597-603 (2005).

31 Wang, S. et al. ARF-mediated SUMOylation of Apak antagonizes ubiquitylation and promotes its nucleolar accumulation to inhibit $47 \mathrm{~S}$ pre-rRNA synthesis. $J$ Mol Cell Biol 7, 154-167 (2015).

32 Ling, Y. et al. Modification of de novo DNA methyltransferase 3a (Dnmt3a) by SUMO-1 modulates its interaction with histone deacetylases (HDACs) and its capacity to repress transcription. Nucleic Acids Res 32, 598-610 (2004).

33 Desterro, J. M., Rodriguez, M. S. \& Hay, R. T. SUMO-1 modification of IkappaBalpha inhibits NF-kappaB activation. Mol Cell 2, 233-239 (1998).

34 Vertegaal, A. C. et al. Distinct and overlapping sets of SUMO-1 and SUMO-2 target proteins revealed by quantitative proteomics. Mol Cell Proteomics $\mathbf{5}$, 2298-2310 (2006).

35 Campagna, M. et al. SIRT1 stabilizes PML promoting its sumoylation. Cell Death Differ 18, 72-79 (2011).

36 Gonzalez-Santamaria, J. et al. Regulation of the tumor suppressor PTEN by SUMO. Cell Death Dis 3, e393 (2012).

37 Marcos-Villar, L. et al. Kaposi's sarcoma-associated herpesvirus protein LANA2 disrupts PML oncogenic domains and inhibits PML-mediated transcriptional repression of the survivin gene. J Virol 83, 8849-8858 (2009). 
A

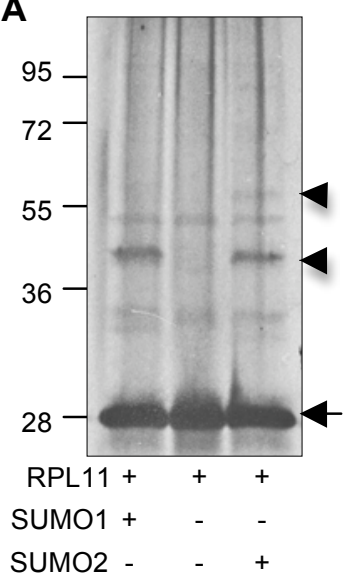

SUMO2 - - +

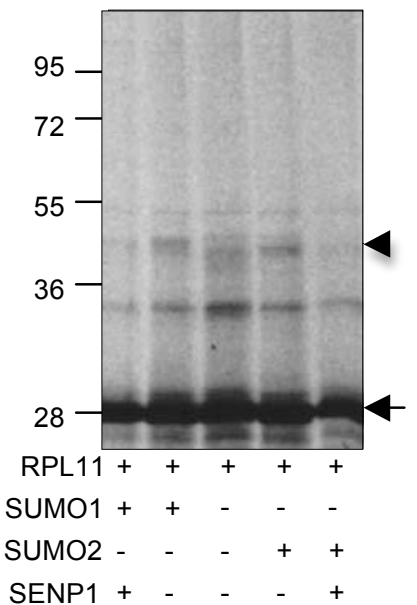

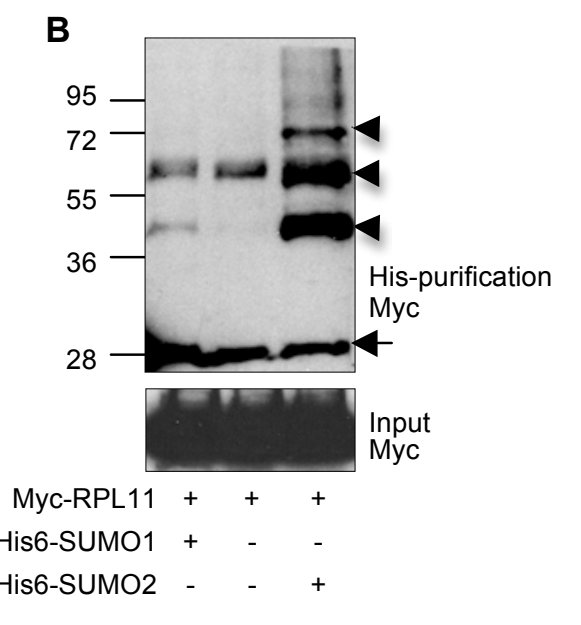

B

Myc-RPL11 + +

His6-SUMO2 - - +

C

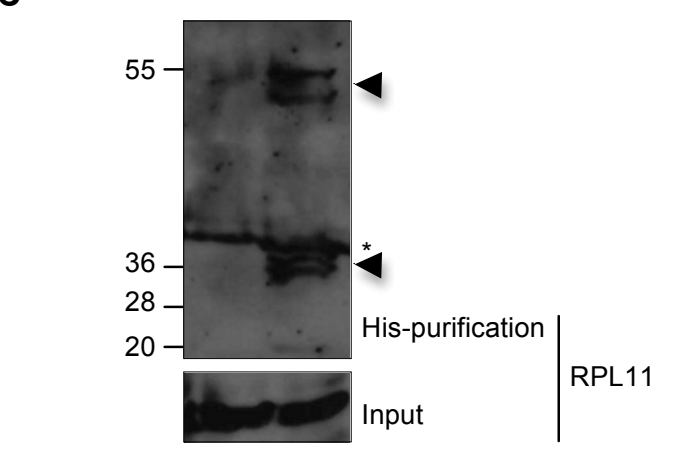

His6-SUMO1 - +

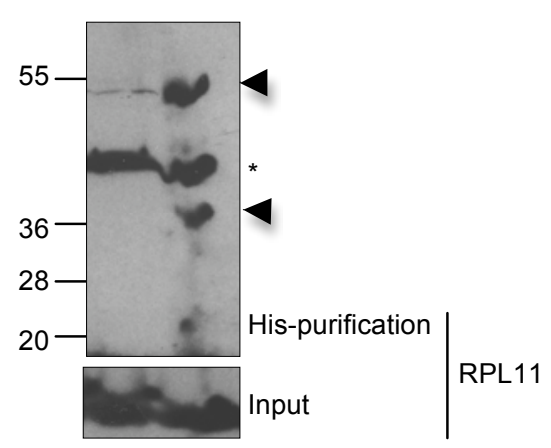

His6-SUMO2 - + 


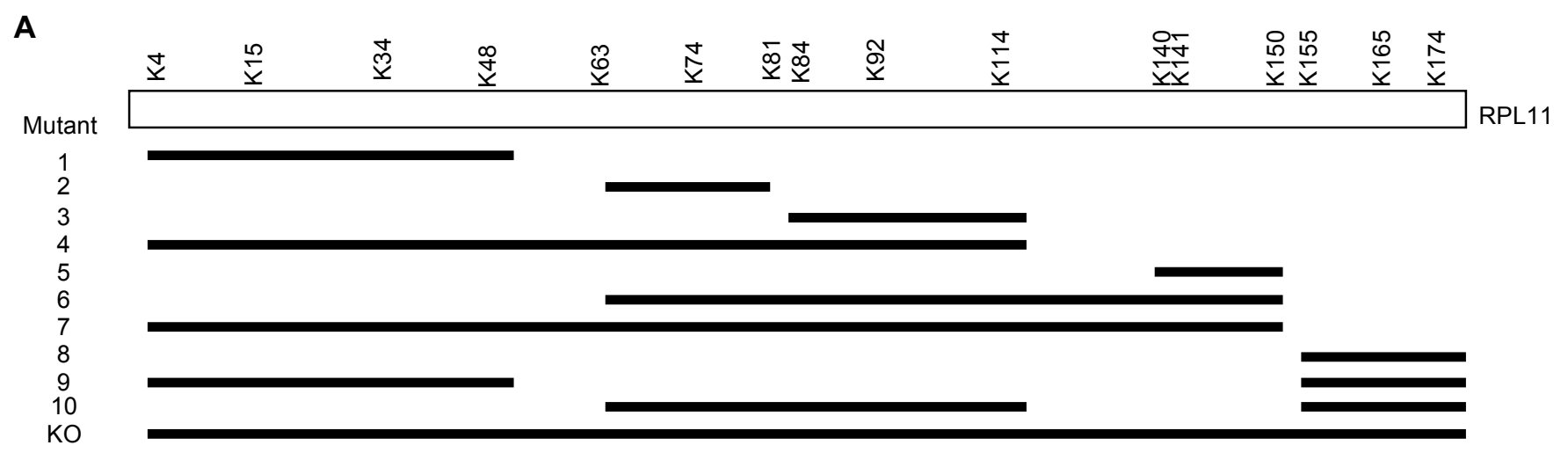

B
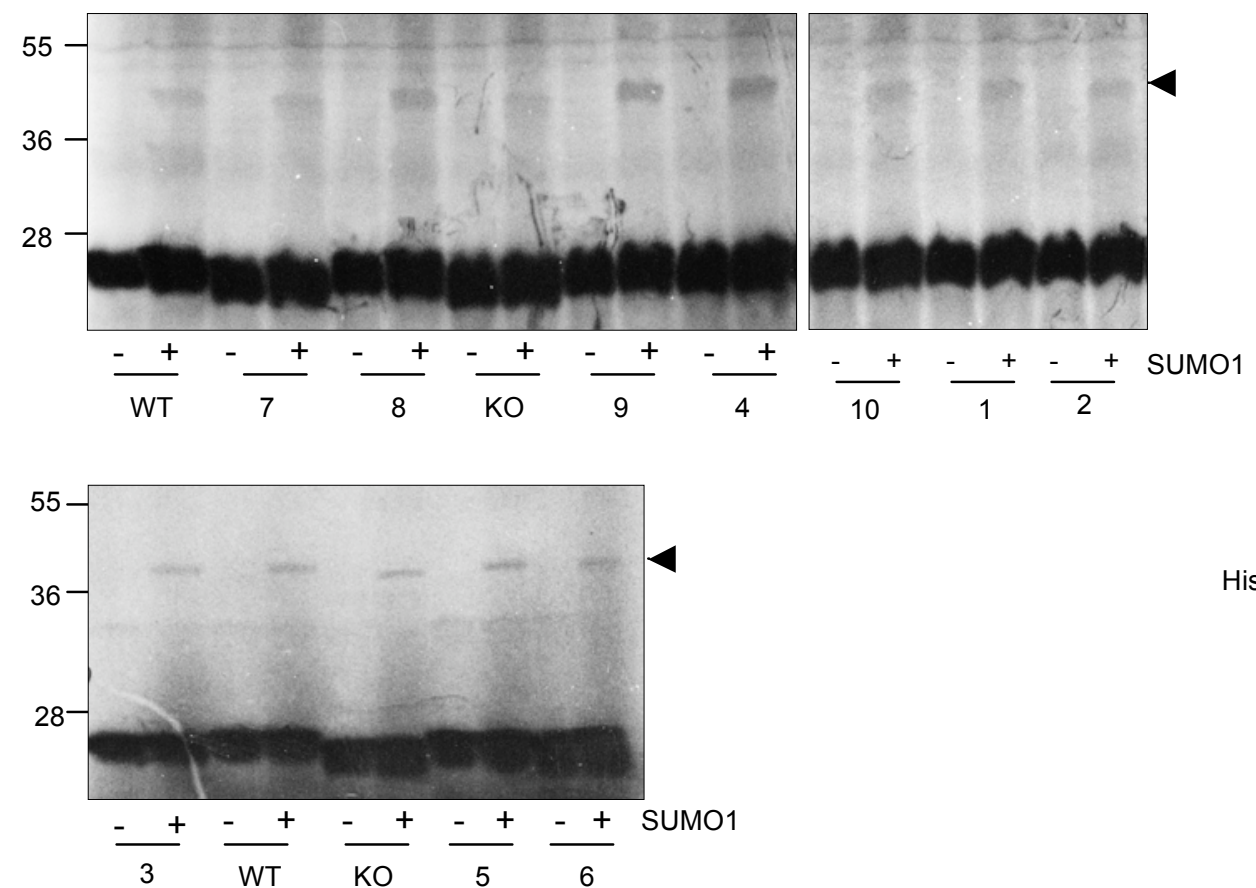

C
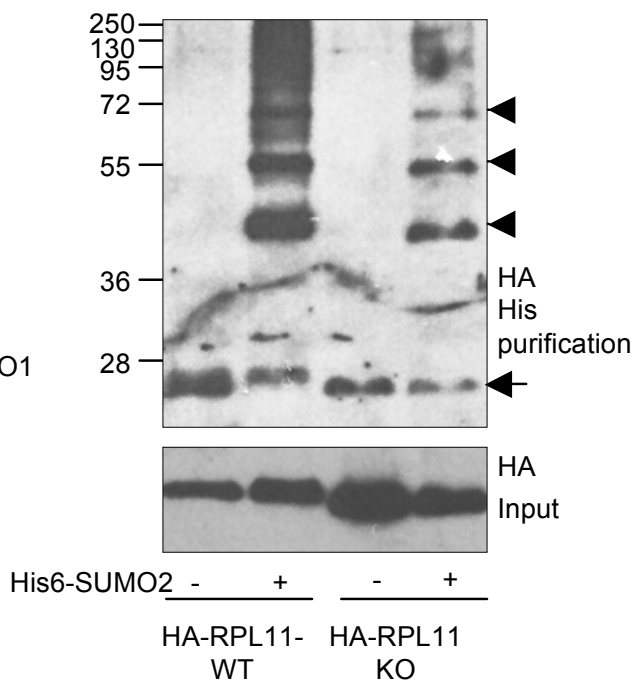

Input 

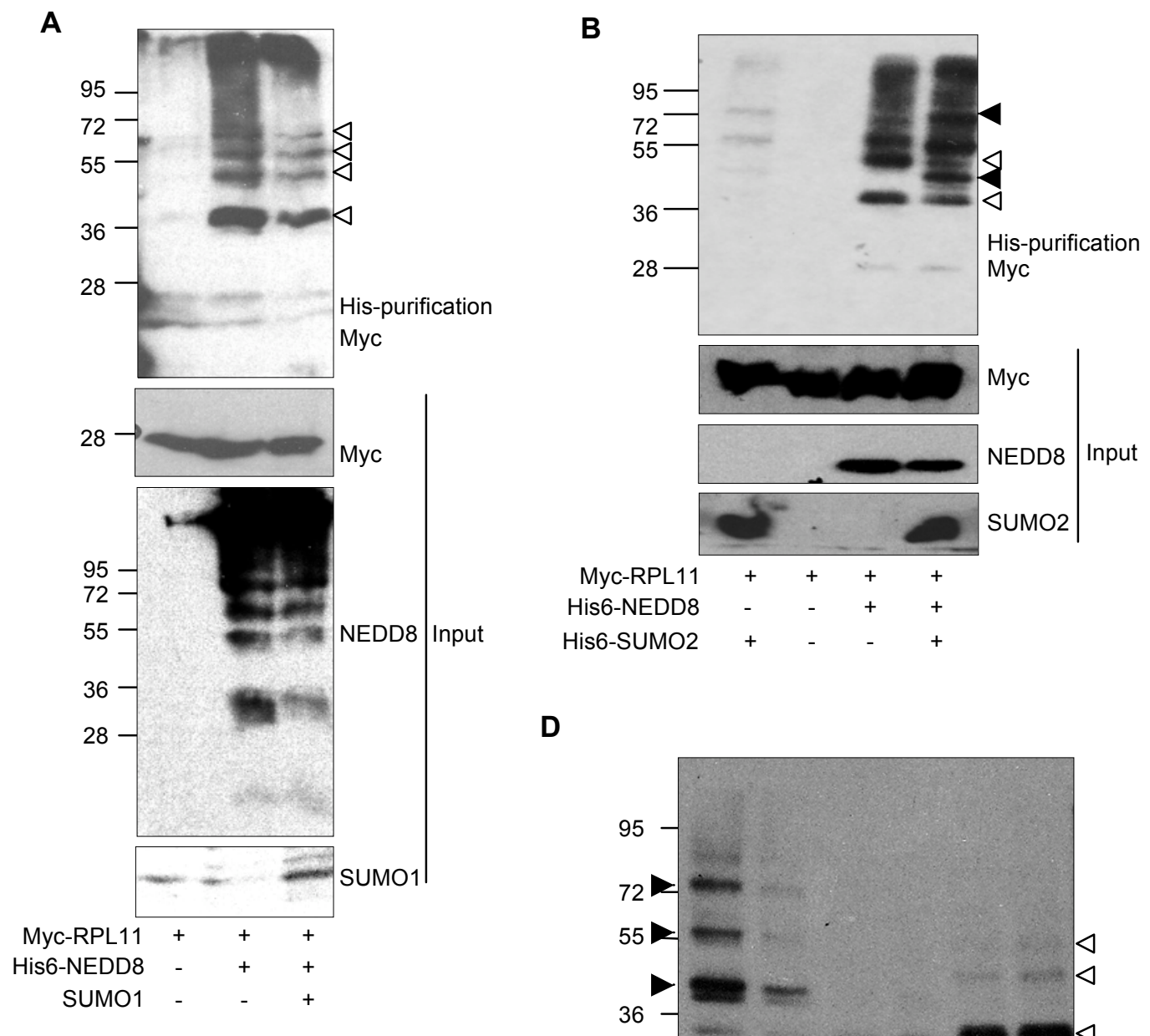

C
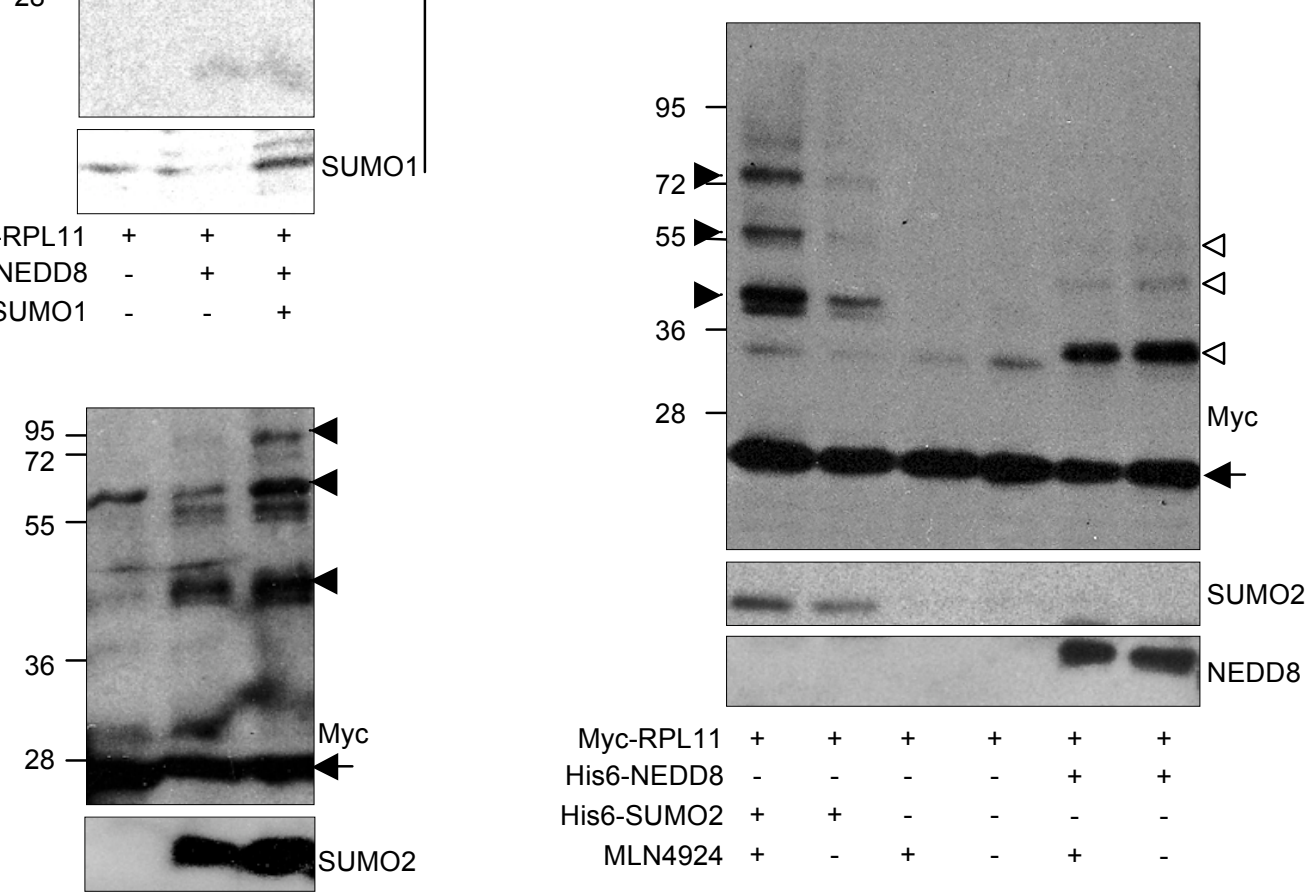

Myc-RPL11 + + + His6-SUMO2 - + + GA - + -

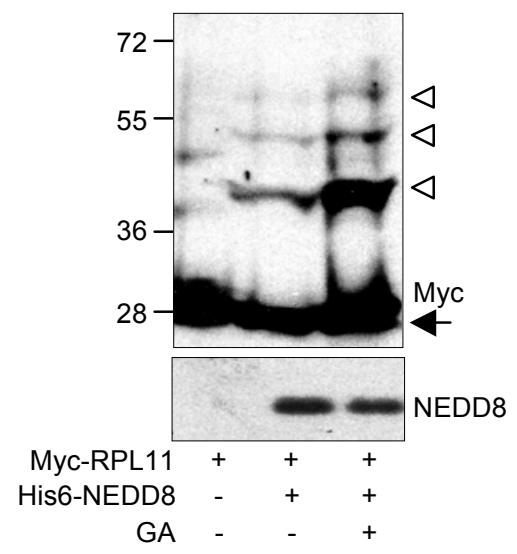



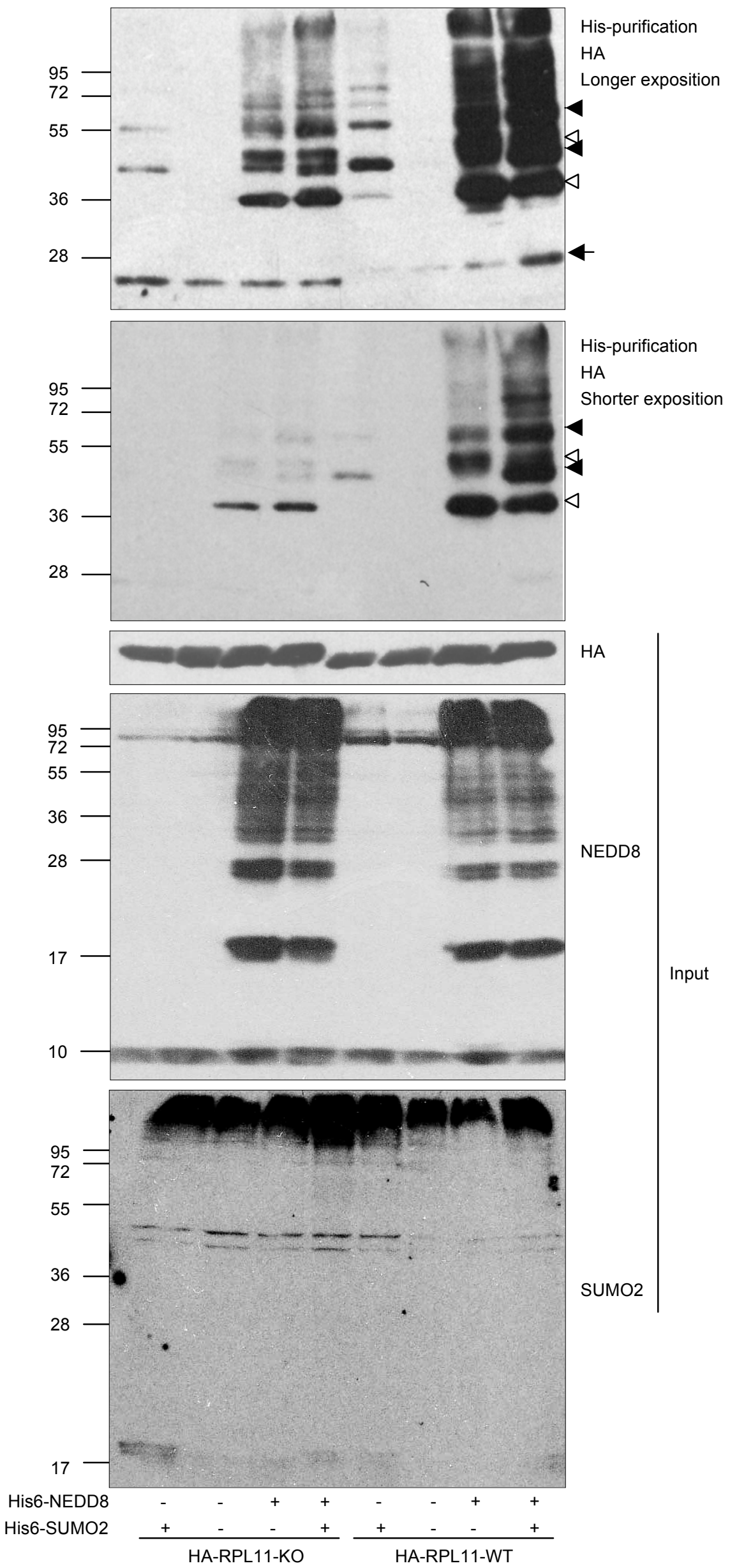


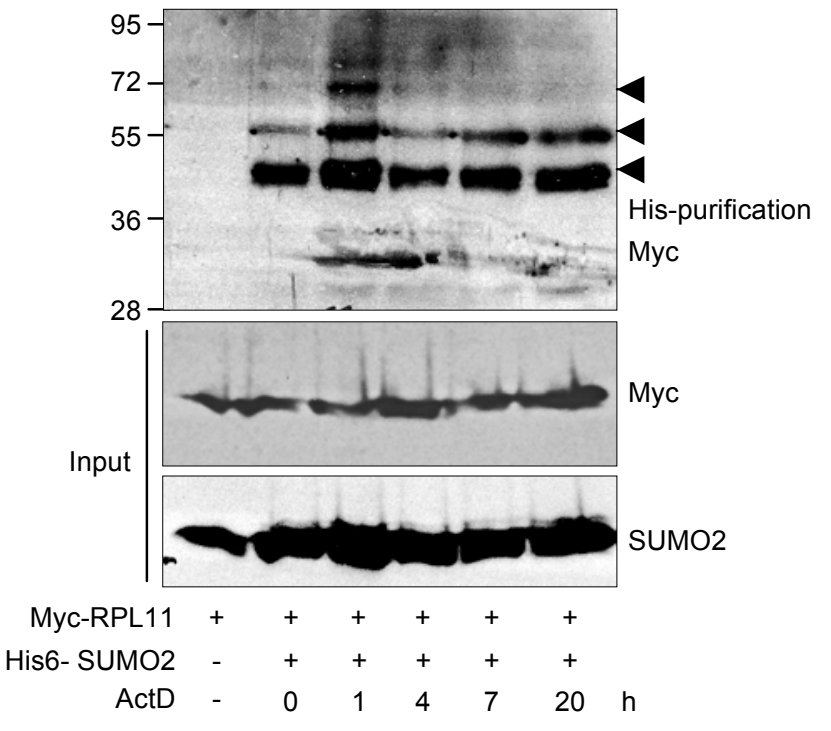



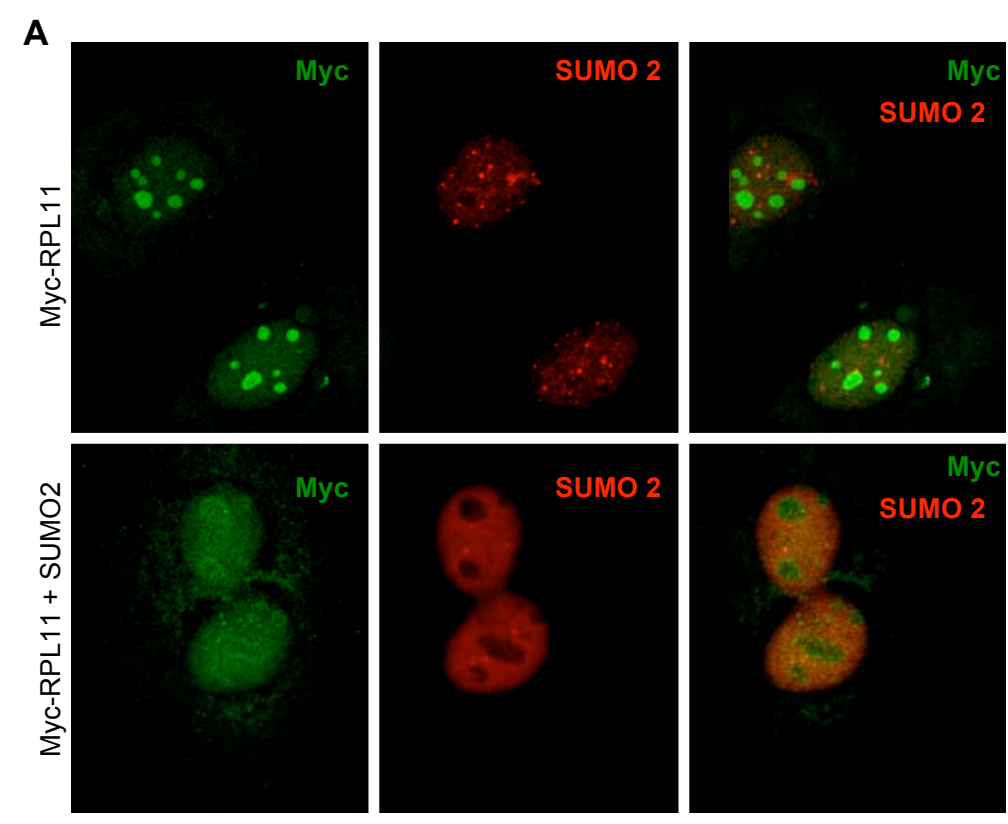

C

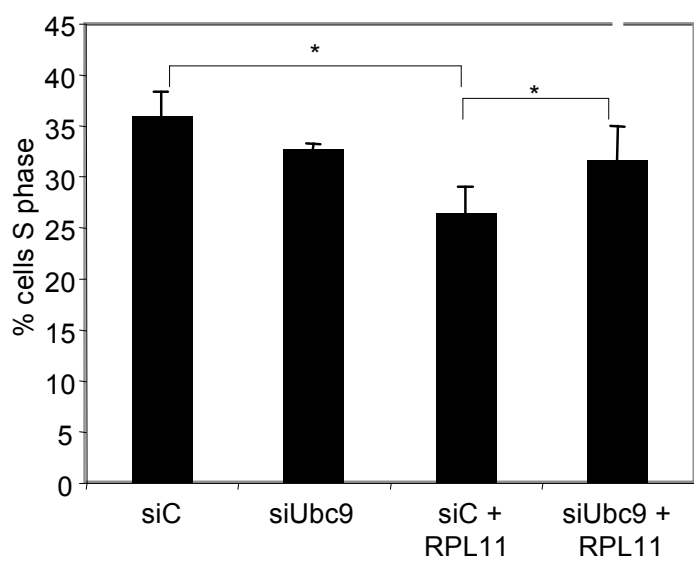

B
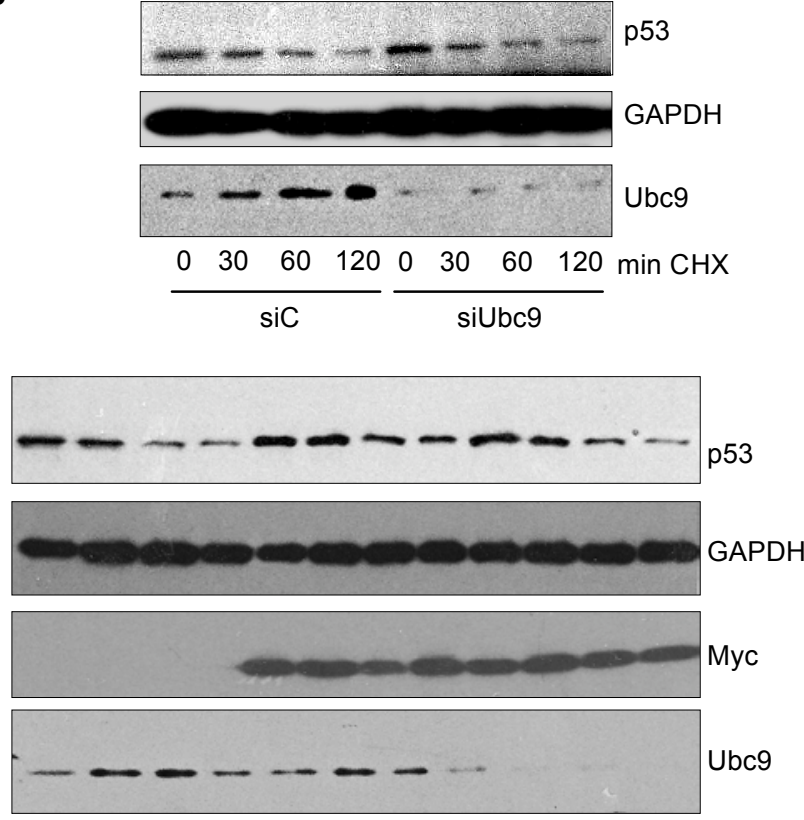

$\frac{0 \quad 30 \quad 60120}{\text { siC }} \frac{030 \quad 60120}{\begin{array}{c}\text { siC+ } \\ \text { myc-RPL11 }\end{array}} \frac{030 \quad 60120}{\begin{array}{c}\text { siUbc9+ } \\ \text { myc-RPL11 }\end{array}} \operatorname{min~CHX}$ 
A

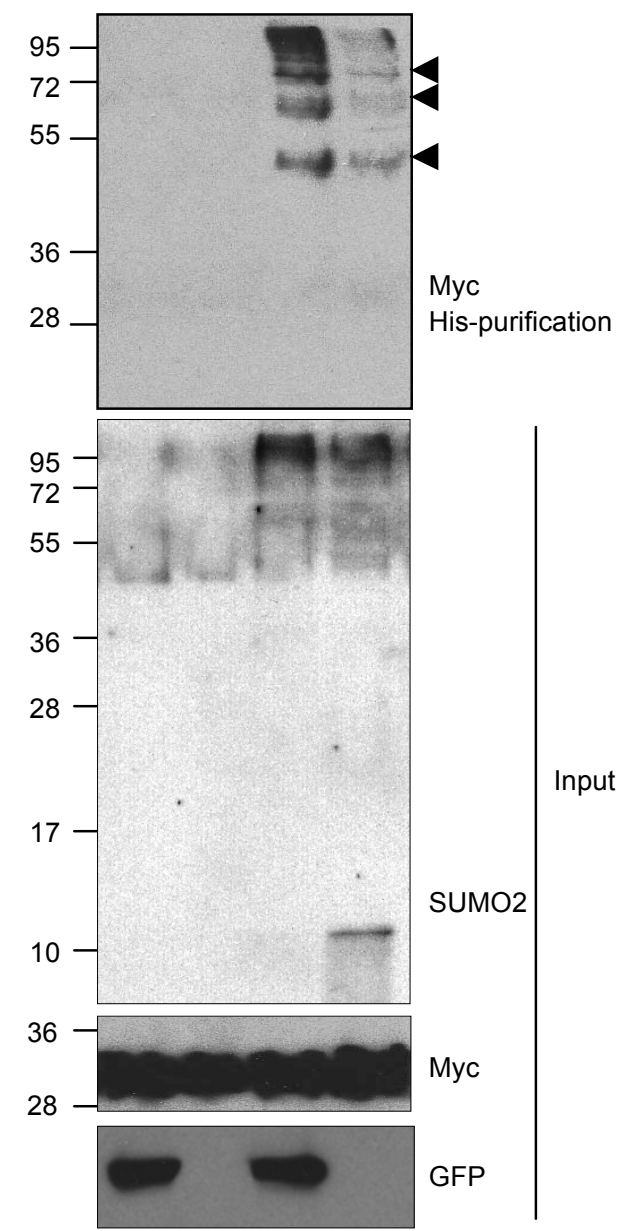

Myc-RPL11 + + + +

His6-SUMO2 - -++

GFP-ARF + - + -

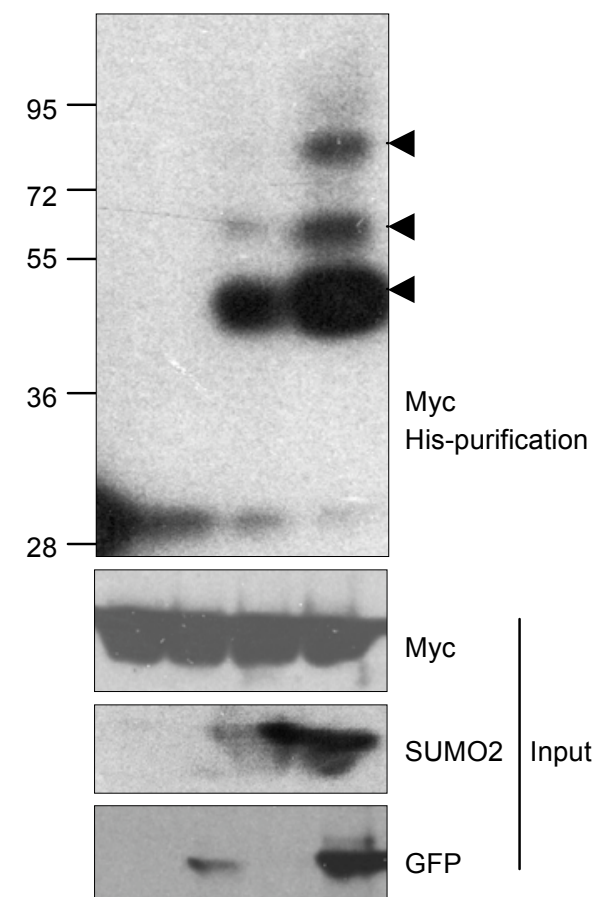

Myc-RPL11 + + + +

His6-SUMO2 - -++

GFP-ARF - + - +
B

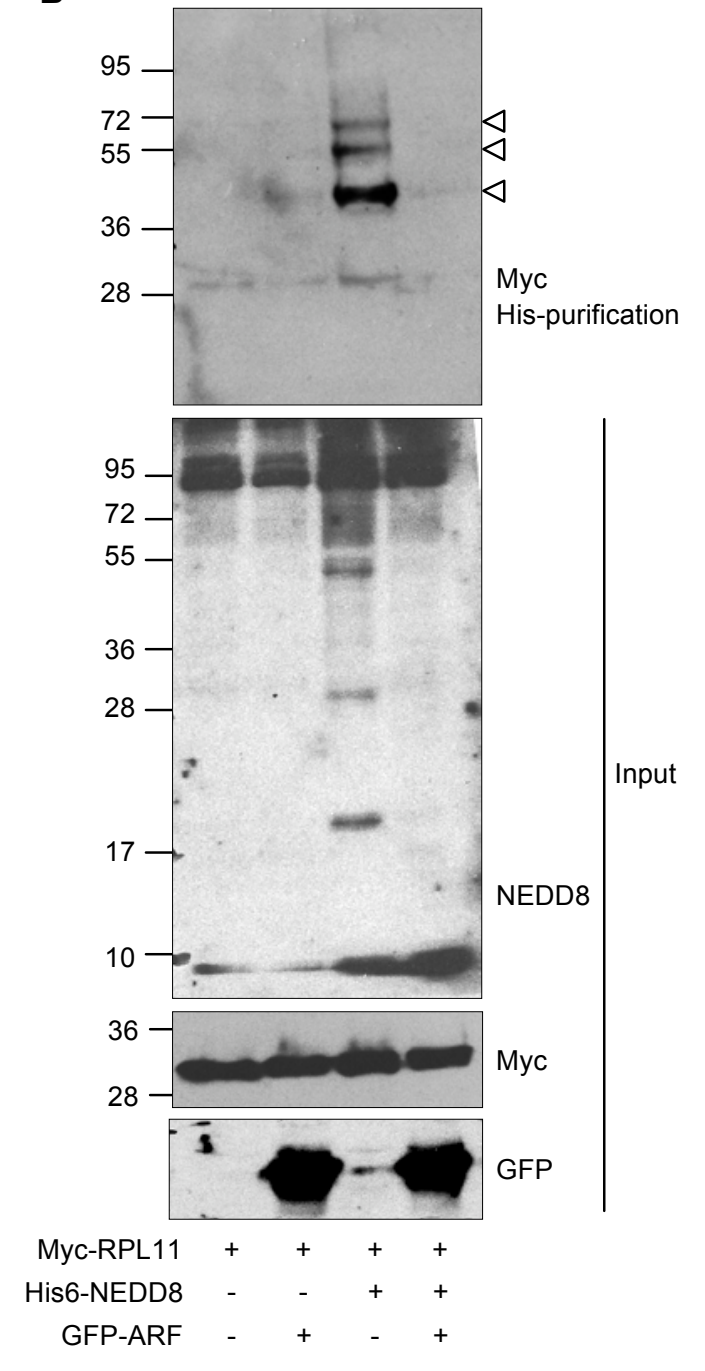

